\title{
Tuning of Predictive Controllers for Drinking Water Networked Systems *
}

\author{
Rodrigo Toro* Carlos Ocampo-Martínez* Filip Logist** \\ Jan Van Impe ** Vicenç Puig* \\ * Institut de Robòtica i Informàtica Industrial, CSIC-UPC \\ Llorens i Artigas 4-6, 08028 Barcelona, Spain \\ \{rtoro, cocampo, vpuig\}@ iri.upc.edu \\ ** BioTeC \& OPTEC, \\ Department of Chemical Engineering, K.U. Leuven, Leuven, Belgium \\ $\{$ filip. logist, jan.vanimpe\}@cit.kuleuven.be
}

\begin{abstract}
In this paper, two tuning strategies for a multi-objective predictive controller applied to a drinking water network (DWN) are proposed. A control-oriented DWN model is briefly reviewed, together with its management objectives. A comparison of methods to explore the Pareto front of the multi-objective optimisation (MOO) problem behind the predictive controller is presented with an effective normalisation method for the model predictive control (MPC) objectives. The proposed tuning strategies, applied to a real-life case study, are compared. Finally, simulation results show that the proposed MPC tuning strategies outperform the baseline results.
\end{abstract}

Keywords: Predictive control, large-scale systems, drinking water networks, optimization, pareto-optimal sets

\section{INTRODUCTION}

The tuning task for model predictive control (MPC) laws has been widely investigated (see Garriga and Soroush (2010)), specially for industrial implementations of this control strategy. In Wojsznis et al. (2003), a practical tuning approach is presented, with some guidelines to adjust the basic MPC parameters, giving special emphasis in the modelling tasks. In Long and Gatzke (2005) and Gatzke and Doyle III (2001), mixed-integer methods, stated in Tyler and Morari (1999) (specifically propositional logic), are used to discretise and prioritise control objectives in an MPC strategy. One advantage of methods based on propositional logic is that control objectives are explicitly stated and prioritised, thus avoiding some uncertainties associated with MPC controller tuning. In Kerrigan and Maciejowski (2002), a class of objective functions, that can be incorporated into a prioritised, multi-objective optimisation problem, for which a solution can be obtained by solving a sequence of single-objective, constrained, convex programming problems is presented. The main disadvantage of these methods is the computational cost to solve the optimisation problems online.

\footnotetext{
* Work of R. Toro, C. Ocampo-Martinez and V. Puig has been supported by the Spanish research project WATMAN (CICYT DPI2009-13744) of the Science and Technology Ministry, the DGR of Generalitat de Catalunya (SAC group Ref. 2009/SGR/1491) and the EU project WIDE (FP7-IST-224168). The authors specially thank the support received from AGBAR in the case study of this paper.

Work of F. Logist and J.F.M. Van Impe is supported in part by Projects OT/09/025/TBA, OT/10/035, OPTEC (Center-of-Excellence Optimization in Engineering) PFV/10/002 and SCORES4CHEM KP/09/005 of the Katholieke Universiteit Leuven, and by the Belgian Program on Interuniversity Poles of Attraction, initiated by the Belgian Federal Science Policy Office. J.F.M. Van Impe holds the chair Safety Engineering sponsored by the Belgian chemistry and life sciences federation essenscia.
}

In the current paper, two methods for tuning an MPC controller for a large-scale system, particularly a drinking water network (DWN), are proposed. The first is based on a histogram of the weight combinations, and the second is based on a model between the average water demand and a weight combination index. The MPC controller referred in this work takes into account a forecast of water demands that incorporates the hourly and daily seasonalities. This forecast has been introduced into the MPC prediction model in order to enrich the information of the disturbances behaviour over the prediction horizon.

One of the main objectives of this work is to explore the optimal, non-dominated solution space of the multiobjective predictive control strategy applied to the DWN system, i.e., the Pareto front of the problem, and choose a solution in line with the management objectives. The aim behind the Pareto front calculation is to find a direct relation between the weights of the solution points and the water demands - which are measured disturbances for the control problem - in order to state an adaptive tuning strategy for the online MPC implementation.

The two main contributions of this paper are the following. First, it is highlighted that the Pareto front of the DWN control problem is not static due to the disturbances changing the control problem constantly. Hence, it is necessary to adjust the controller continually. Second, it is noted that the tuning of the controller is explicitly related to the disturbances of the DWN problem, i.e., water demands.

The outline of this work is the following: in Section 2, the DWN control problem is stated; in Section 3, methods to 
calculate the Pareto front of a DWN predictive controller are presented. In Section 4, two strategies to tune the MPC weighting factors are proposed; later, in Section 5, simulation results are presented and discussed. Finally, in Section 6, comments and conclusions are provided.

\section{DRINKING WATER NETWORK CONTROL PROBLEM}

The control-oriented modelling principles of DWNs have been widely presented in the literature (see Brdys and Ulanicki (1994)). In order to obtain the DWN controloriented model, the constitutive elements and basic relationships are introduced in this section (see OcampoMartinez et al. (2009)).

\subsection{DWN Components and Control Variables}

Water Storing Tanks. Water tanks are modelled using a mass balance expression related with the stored water volume, inflows and outflows. For the $i$-th tank, the discrete time expression is

$$
x_{i}(k+1)=x_{i}(k)+\Delta t\left(\sum q_{i n, i}(k)-\sum q_{\text {out }, i}(k)\right),
$$

where $x_{i}(k)$ is the volume of the $i$-th tank, at time $k \in \mathbb{Z}_{+}$, in $\mathrm{m}^{3} ; \Delta t$ is the sampling time in seconds; $q_{i n, i}(k)$ is the inflow to the $i$-th tank at time $k$ in $\mathrm{m}^{3} / \mathrm{s}$; and $q_{\text {out }, i}(k)$ is the outflow flow from the $i$-th tank at time $k$ in $\mathrm{m}^{3} / \mathrm{s}$.

Inflows are treated as manipulated variables. Outflows are modelled as manipulated variables or measured disturbances (sector of consume).

The model of a tank includes the physical constraint

$$
x_{i}^{\min } \leq x_{i}(k) \leq x_{i}^{\max }
$$

where $x_{i}^{\text {min }}$ and $x_{i}^{\max }$ are given in $\mathrm{m}^{3}$.

Valves and Pumps. In order to control the water flow, two kind of actuators are considered: valves and pumps. In order to control water flows, setpoints of the regulatory flow control loops are taken as manipulated variables, denoted as $u$. Both actuators have physical constraints, which are expressed as

$$
u_{i}^{\min } \leq u_{i}(k) \leq u_{i}^{\max }
$$

where $u_{i}^{\min }$ and $u_{i}^{\max }$ are given in $\mathrm{m}^{3} / \mathrm{s}$.

In terms of economic costs, it is more expensive to control water flows with pumping stations than with valves.

Network Nodes. Nodes are points of the network where water flows are merged or split. They are represented as mass balance relations and are modelled as equality constraints of inflows and outflows of the nodes in the following way

$$
\sum q_{\text {in }, i}(k)=\sum q_{\text {out }, i}(k) .
$$

Sectors of Consume. Sectors of consume represent the water demand made by DWN users of a specific geographical area. These demands are modelled as known system disturbances. In this application, a demand forecast module is used together with the MPC controller.

\subsection{DWN Linear Model}

Considering the above expressions, a linear state-space model for control purposes has been stated as follows

$$
\begin{gathered}
\mathbf{x}(k+1)=A \mathbf{x}(k)+B \mathbf{u}(k)+B_{p} \mathbf{d}(k), \\
E \mathbf{u}(k)+E_{d} \mathbf{d}(k)=0,
\end{gathered}
$$

where $\mathbf{x}(k) \in \mathbb{R}^{n}$ is the state vector of water volumes corresponding to the $n$ tanks. All the states are supposed to be measurable; $\mathbf{u}(k) \in \mathbb{R}^{m}$ is the vector of manipulated flows through the $m$ actuators; $\mathbf{d}(k) \in \mathbb{R}^{p}$ is the vector of demands acting as measured disturbances for the $p$ sectors of consume; $A, B$ and $B_{p}$ are state-space system matrices; and $E$ and $E_{d}$ are matrices of suitable dimensions that describe the mass balance relation at network nodes.

\subsection{DWN Control Objectives}

Water Production and Transport Cost. Main economic costs associated to drinking water production are due to chemicals (used in water treatment), legal canons (taxes), and electricity costs. The total cost associated to water transportation is

$$
f_{1}(k)=\left(\alpha_{1}+\alpha_{2}(k)\right) \mathbf{u}(k) \Delta t,
$$

where $\mathbf{u}(k)$ is a vector of control actions at time $k ; \alpha_{\mathbf{1}}$ is a known vector related to economic costs of water treatment; and $\alpha_{\mathbf{2}}(k)$ is a known time-varying vector associated to the economic cost of water flows related to pumping stations. The time dependence is given by the electric pumping cost, which varies along the time.

Safety Storage Term. The objective of this function is to penalise quadratically the predicted volumes that go down from a pre-established value. Hence, the decision vector includes those penalisation variables (denoted as $\varepsilon$ ), see Adrian (2010) for further details.

The objective function has been defined as

$$
f_{2}(k)=\varepsilon(k)^{\top} \varepsilon(k),
$$

where $\varepsilon(k)$ is the amount of soft constraint violation. $\varepsilon$ has been defined as when there is no violation $\varepsilon=0$.

Smoothness Objective. It is desirable to avoid excessive variations in the control actions, given the flow-based nature of the system model. Pumping stations and valves should operate smoothly avoiding big "steps" in the pressurised pipes. This quadratic term is expressed as

$$
f_{3}(k)=\Delta \mathbf{u}(k)^{\top} \Delta \mathbf{u}(k),
$$

where $\Delta \mathbf{u}(k)$ is the vector of control signal variations, defined as $\Delta \mathbf{u}(k) \triangleq \mathbf{u}(k)-\mathbf{u}(k-1)$.

\subsection{Optimisation Problem Statement}

The multi-objective optimisation (MOO) problem behind the MPC of the DWN is

$$
\mathrm{MPC}: \min _{\mathcal{Z}} \gamma_{1} F_{1}(\mathcal{Z})+\gamma_{2} F_{2}(\mathcal{Z})+\gamma_{3} F_{3}(\mathcal{Z})
$$

subject to

$$
\begin{aligned}
& \Omega \mathcal{Z} \leq \omega, \text { and } \\
& H \mathcal{Z}=h,
\end{aligned}
$$

where $\mathcal{Z}=\left[\begin{array}{ll}\Delta \mathcal{U} & \varepsilon\end{array}\right]^{\top}$ is the vector of decision variables, $\Delta \mathcal{U}$ is the vector of control variations, $\Delta \mathbf{u}$, and $\varepsilon$ is the 
Table 1. Pareto Front Calculation Methods and MPC

\begin{tabular}{|c|c|c|c|c|}
\hline Method & $\begin{array}{r}\mathrm{M} \\
\text { Weights } \\
\text { Implicit }\end{array}$ & $\begin{array}{l}\text { Relation } \\
\text { Explicit }\end{array}$ & Pros & Cons \\
\hline NNC & $\sqrt{ }$ & $\mathrm{x}$ & $\begin{array}{l}\text { - Front } \\
\text { exploration }\end{array}$ & $\begin{array}{l}\text { - Function } \\
\text { normalisation } \\
\text { - CPU time } \\
\text { - Convexity of } \\
\text { subproblems }\end{array}$ \\
\hline ENNC & $\sqrt{ }$ & $\sqrt{ }$ & $\begin{array}{l}\text { - Front } \\
\text { exploration } \\
\text { - Function } \\
\text { normalisation }\end{array}$ & $\begin{array}{l}\text { - CPU time } \\
\text { - Convexity of } \\
\text { subproblems }\end{array}$ \\
\hline ENNCP & $\sqrt{ }$ & $\mathrm{x}$ & $\begin{array}{l}\text { - Front } \\
\text { exploration } \\
\text { - Function } \\
\text { normalisation }\end{array}$ & $\begin{array}{l}\text { - CPU Time } \\
\text { - Convexity of } \\
\text { subproblems }\end{array}$ \\
\hline NWS & $\sqrt{ }$ & $\sqrt{ }$ & $\begin{array}{l}\text { - CPU time } \\
\text { - Preserve } \\
\text { convexity }\end{array}$ & $\begin{array}{l}\text { - Front } \\
\text { exploration }\end{array}$ \\
\hline
\end{tabular}

vector of tank volume constraint violation, both of them extended over the prediction horizon; $F_{1}, F_{2}$, and $F_{3}$ are the extension of objective functions (6), (7), and (8) for the MPC formulation (see Maciejowski (2001)). Note that $F_{1}$ is linear while $F_{2}$ and $F_{3}$ are quadratic functions; and $\gamma_{1}, \gamma_{2}$, and $\gamma_{3}$ are weighting factors of the optimisation problem behind the MPC. These weighting factors are the MPC tuning parameters studied in this paper.

Constraints (9b) and (9c) are an extension of the physical limits and mass balances at network nodes expressed in (2), (3), and (5b).

\section{PARETO FRONT CALCULATION OF A MOO PROBLEM}

Efficient approaches to generate the Pareto front for multiobjective optimal control problems have been recently reported (see, e.g., Logist et al. (2009, 2010)).

In this section, four methods to calculate the Pareto front of a MOO problem are compared. Three of them are based on the normalised normal constraints (NNC) idea presented in Messac et al. (2003). The fourth is a normalised version of the well known weighted sum method (see Ding et al. (2006), and Marler and Arora (2010) for details).

Table 1 shows a comparison between the tested Pareto front calculations methods. They are the NNC, the enhanced NNC (ENNC) and a variation of it using pseudo anchor points (ENNCP), these two latter presented in Sanchis et al. (2008). Moreover, the normalised weighted sum (NWS) method, that takes advantage of the normalisation scheme proposed in the ENNCP, is also compared.

The NWS method has a direct relation between Pareto front points and weighting factors. Recently, an explicit relation between Pareto front points calculated with the ENNC method and weighting factors have been stated (see Logist and Van Impe (2010); Logist et al. (2011)).

\subsection{Objective Function Normalisation}

In the normalised design space, it is desired that the anchor points, that correspond to the best possible values for respective individual objectives (see Sanchis et al. (2008)), have the following coordinates:

$$
\begin{aligned}
\bar{F}^{1 *} & =\left[\begin{array}{llllll}
0 & 1 & 1 & \ldots & 1 & 1
\end{array}\right]^{\top}, \\
\bar{F}^{2 *} & =\left[\begin{array}{llllll}
1 & 0 & 1 & \ldots & 1 & 1
\end{array}\right]^{\top}, \\
\vdots & \\
\bar{F}^{q *} & =\left[\begin{array}{llllll}
1 & 1 & 1 & \ldots & 1 & 0
\end{array}\right]^{\top},
\end{aligned}
$$

where $\bar{F}^{i *}, i=1 \ldots q$, are the normalised anchor points of the $q$ objective functions.

In general, when the MOO problem presents "disparate" objectives scales, condition (10) will not always be fulfilled and the normalised objective space will not be preserved. For this reason, a solution with an exact linear transformation between the anchor points and the normalised ones is proposed in the ENNCP method.

In order to normalise the MOO problem, the following steps must be fulfilled:

(1) Calculate the difference matrix $\Psi$

$$
\Psi=F^{* *}-\left[\begin{array}{lll}
F^{u} & \ldots & F^{u}
\end{array}\right]_{q \times q}
$$

where

- $F^{* *}$ is a matrix with the $q$ pseudo anchor points, defined as

$$
F^{* *}=\left[\begin{array}{llll}
F_{1}^{u} & F_{1}^{N} & \ldots & F_{1}^{N} \\
F_{2}^{N} & F_{2}^{u} & \ldots & F_{2}^{N} \\
& \vdots & \ddots & \\
F_{q}^{N} & F_{q}^{N} & \ldots & F_{q}^{u}
\end{array}\right]_{q \times q} ;
$$

- $F_{i}^{u}$ is the $i$-th value of the utopia point vector $F^{u}$, of dimension $q \times 1$, which corresponds to the best possible value for the $i$-th objective; and

- $F_{i}^{N}$ is the $i$-th value of the nadir point vector, which corresponds to the worst possible value for the $i$-th objective.

(2) Calculate the linear transformation matrix $T$

$$
T=\left[\begin{array}{cccc}
0 & 1 & \ldots & 1 \\
1 & 0 & \ldots & 1 \\
\vdots & \vdots & \ddots & \vdots \\
1 & 1 & \ldots & 0
\end{array}\right] \Psi^{-1} ; \text { and }
$$

(3) Calculate the normalised objective functions

$$
\bar{F}=T\left(F-F^{u}\right),
$$

where $F$ is the unnormalised vector of objective functions.

Remark 3.1. Condition (10) applied to the pseudo anchor points, $F^{* *}$, will always be fulfilled in spite of disparate objectives scales.

Remark 3.2. Matrix T, calculated with pseudo anchor points, will always be diagonal with elements

$$
t_{j j}=\frac{1}{F_{j}^{N}-F_{j}^{u}} ; \quad \forall j \in 1, \ldots, q .
$$

\subsection{Normalised Weighted Sum}

In the Normalised Weighted Sum (NWS) method, each point is defined as

$$
\bar{J}^{i}=\mathbf{w}_{i}^{\top} \bar{F},
$$

with the condition

$$
\sum_{j=1}^{q} w_{i}(j)=1,
$$




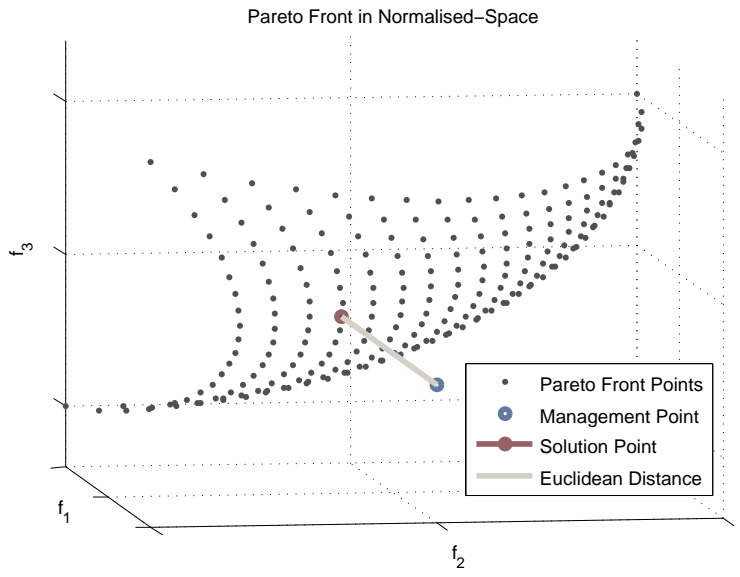

Fig. 1. Decision making strategy graphically explained.

where $\mathbf{w}_{i}$, denotes a vector of $p$ weights, and $\bar{F}$ is the vector of normalised functions calculated by using the approach previously presented and $w_{i}(j), j \in 1, \ldots, q$, is the $j$-th element of the $\mathbf{w}_{i}$ vector.

\section{DECISION MAKING AND TUNING STRATEGIES}

\subsection{Decision Making Based on a Management Point}

Picking a desirable point out of the Pareto optimal solutions involves a decision maker. The decision maker is a person who has insights into the problem and who is able to express preference relations between different solutions. The task of the decision maker can be automated using a decision making algorithm.

A decision making (DM) strategy, based on the minimum distance to a point over the normalised design space, is proposed. The main idea is to define this point, from now on called the Management Point (MP), and calculate the minimum Euclidean distance from the solutions of the Pareto front and the MP. The selected solution is calculated in the following way:

$$
X_{M P}=\arg \min \left\|\bar{J}^{i}-\mathrm{MP}\right\|, \quad i=1 \ldots r,
$$

where $\bar{J}^{i}$ is the $i$-th point of the obtained Pareto front. An example could be the point $\left[\begin{array}{lll}0 & 0 & 0\end{array}\right]^{\top}$, supposed to yield the best trade-off for equality among the objectives.

In order to establish a prioritisation scheme, an MP based on prioritisation percentages $(\mathrm{PP})$ has been defined as mathrmMP $=\left[\begin{array}{llll}m p_{1} & m p_{2} & \ldots & m p_{q}\end{array}\right]^{\top}$, where $m p_{i}$ is the $i$-th coordinate of the MP, defined as (100$\left.p p_{i}\right) \bar{F}_{i}^{N} / 100 ; p p_{i}$ is the priority percentage of the objective function $i$ (100 is the maximum priority percentage), defined by the user as mathrmPP $=\left[\begin{array}{llll}p p_{1} & p p_{2} & \ldots & p p_{q}\end{array}\right]$; and $\bar{F}_{i}^{N}$ is the $i$-th normalised nadir point.

In Fig. 1, a graphical explanation of the decision making scheme is presented. The applied control action corresponds to the solution point, which is the one that has the minimum distance to the MP.

Remark 4.1. The introduced DM strategy was an a posteriori articulation of preferences as a solution point is selected after the calculation of Pareto points (see Marler and Arora (2010)).
Remark 4.2. If this DM strategy is used in an on-line implementation, the calculation time must be taken into account because the computational cost of calculating the Pareto front at each sampling time may be high.

\subsection{Tuning Strategy}

In this section two simple tuning strategies, derived from the DM strategy, are presented.

The main idea is to avoid the calculation of the entire Pareto front at each MPC iteration by creating a model between the weight combination, used in the solution point $\bar{J}^{*}$, and an average of the sectors of demand, $\tilde{d}$.

In the following two subsections, the steps to calculate the MPC weighting factors are described.

\section{Histogram Based Weighting Factors Combination.}

(1) Calculate the Pareto front of the problem using the NWS method;

(2) Using the proposed DM strategy, relate the selected solution points with its corresponding weighting combinations, that is, a column of matrix $W$, which contains all the possible weighting combinations. The columns of matrix $W$ related with the solution points must be stored in a vector of weighting combination indices $\left(\omega_{i d x}\right)$; and

(3) Calculate the histogram of $\omega_{i d x}$ and select the most used weighting combination.

This simple tuning strategy can be used as a starting point for an empirical tuning of MPC weights.

\section{Model Based Weighting Factors Combination.}

(1) Steps (1) and (2) of the previous tuning strategy;

(2) Calculate a regression model of $\omega_{i d x}$ as a function of the average of water demands $(\tilde{d})$; and

(3) Use the regression model from the previous step, from now $\omega_{\text {reg }}(\tilde{d}): \mathbb{R} \rightarrow \mathbb{Z}$, for the calculation of weighting combinations in the online MPC application.

\section{SIMULATION AND RESULTS}

In this section, simulation results are presented. The selected case study is the aggregate model of the Barcelona DWN, see Toro (2010) for details. All the simulations have been done over a time period of three days with a prediction and control horizon of eight hours. The selected sampling time is one hour.

Simulations have been done using the TOMLAB/CPLEX optimisation package for Matlab, and the TomSym modelling tool (see Holmström et al. (2009)).

\subsection{Pareto Front Generation}

The interesting feature about obtaining the Pareto front in an MPC problem is that, at each iteration, the front changes as a function of the disturbances.

Figure 2 shows the obtained Pareto front for the 3-days simulation considering the NWS approach presented in Section 3.2. It can be seen that the obtained Pareto front 

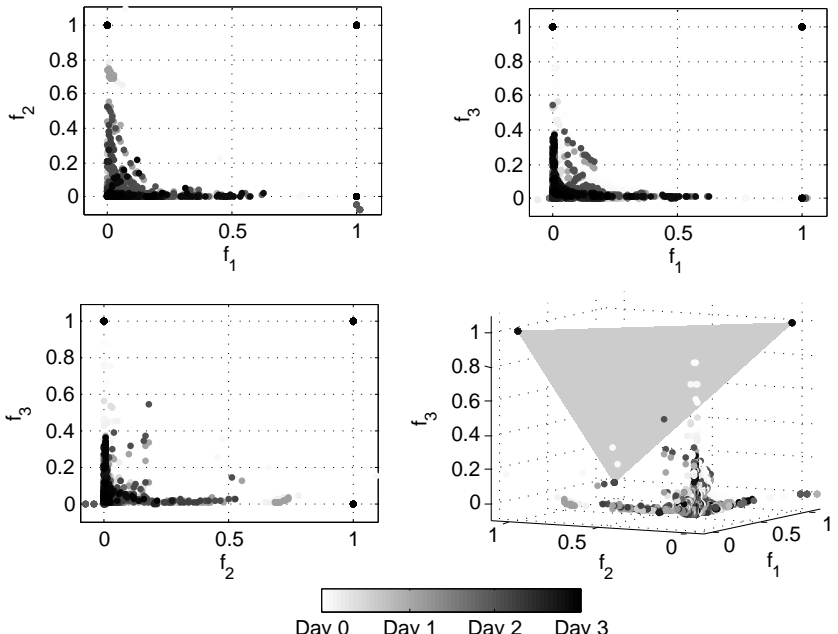

Fig. 2. Pareto Front over the Normalised Design Space obtained with the NWS method.
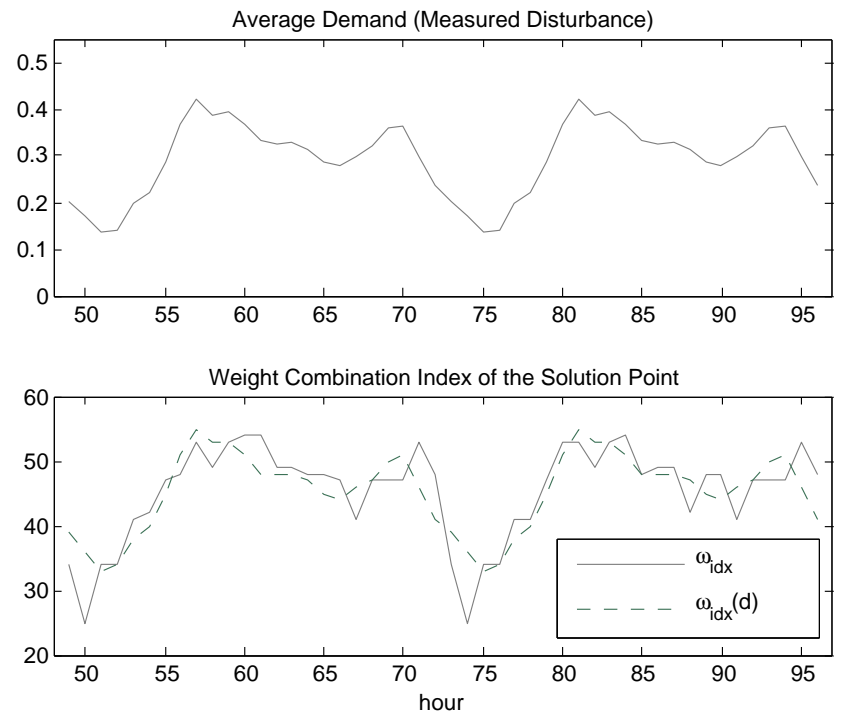

Fig. 3. Temporal Data Plot of $\tilde{d}$ and $\omega_{i d x}$.

is compatible with the proposed DM algorithm, because all the Pareto points are positive. The explored space is notably smaller than the one explored with methods based on normal constraints (see Toro (2010) for further details).

\subsection{Weighting Combinations Model}

In this section, results from the method proposed in Section 3.2 are presented. Figure 3 shows the temporal responses of the weighting combination index, $\omega_{i d x}$, and the average demand, $\tilde{d}$. In Fig. 4, a linear regression between the two variables is shown. The correlation index of $\omega_{i d x}$ and $\tilde{d}$ is 0.83 . Taking into account the results and the correlation between the variables, a sufficiently accurate linear regression model can be calculated.

\subsection{Tuning Comparisons}

Four tuning strategies have been compared for a predictive control scheme over the aggregate model of the Barcelona DWN. In the first place, the original MPC implementation,

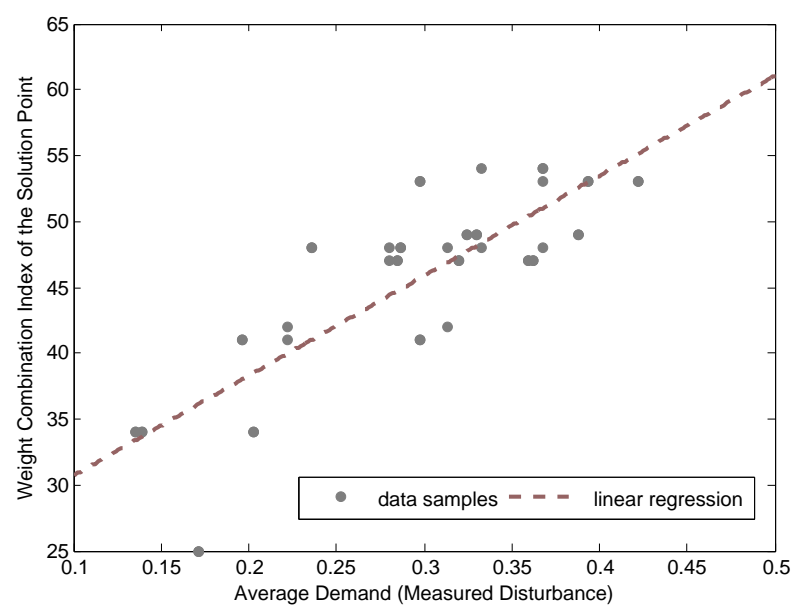

Fig. 4. Linear Regression of $\omega_{i d x}$ and $\tilde{d}$.

without normalisation, is simulated and is used as baseline for comparison purposes. Then, an MPC implementation with normalisation of the objective functions (using the method discussed in Section 3.1), is compared. Next, the histogram-based tuning strategy, and finally, the modelbased tuning scheme results are presented. The first two MPC implementations have been tuned with equitable weighting factors, such that, $\mathbf{w}=\left[\begin{array}{lll}1 / 3 & 1 / 3 & 1 / 3\end{array}\right]^{\top}$.

In order to compare results obtained from the different tuning strategies, three Key Performance Indicators (KPIs) have been defined based on the DWN objective functions. In this section, a brief description of the KPIs is presented.

Economic KPI. This performance indicator is related to the water costs and has been defined based on the Water Production and Transport Cost (6) as

$$
K P I_{€}=\frac{\Delta t}{N} \sum_{k=1}^{N}\left(\alpha_{\mathbf{1}}+\alpha_{\mathbf{2}}(k)\right) \mathbf{u}(k),
$$

where $N$ is the number of samples considered in the evaluation.

Safety KPI. This performance indicator is related to volume-regulation strategy of the tanks. The main idea is to evaluate the mean value of the soft constraint limit violations $\varepsilon$. Therefore, the safety KPI has been defined as

$$
K P I_{\text {safe }}=\frac{1}{N} \sum_{k=1}^{N} \varepsilon(k),
$$

where $\varepsilon(k)$ denotes the amount of the soft constraint violation at time $k$.

Smoothness KPI. This performance indicator is related to the smoothness of control movements and is defined as

$$
K P I_{\Delta u}=\frac{1}{(N-1)} \sum_{k=2}^{N}(\Delta \mathbf{u}(k))^{2},
$$

where $\Delta \mathbf{u}(k)$ is the incremental control movement applied at time $k$.

Remark 5.1. In all the presented KPIs, a reduction of their values implies better results. 
Table 2. Tuning Strategies Comparison

\begin{tabular}{crrrrrr}
\hline \multirow{2}{*}{ Tuning Strategy } & \multicolumn{2}{c}{ Economic KPI } & \multicolumn{2}{c}{ Safety KPI } & \multicolumn{2}{c}{ Smoothness KPI } \\
& Day 2 & Day 3 & Day 2 & Day 3 & Day 2 & Day 3 \\
\hline Original MPC & 34.4477 & 34.5007 & 3921.7 & 3912.3 & 0.0105 & 0.0103 \\
Normalised MPC & 34.5643 & 34.6338 & 3837.6 & 3838.3 & 0.0026 & 0.0025 \\
Histogram-based Weighting & 34.1424 & 34.2004 & 3324.7 & 3337.2 & 0.0017 & 0.0017 \\
Model-based Weighting & 34.1129 & 34.1618 & 3305.1 & 3307.7 & 0.0019 & 0.0019 \\
\hline
\end{tabular}

Table 2 shows the obtained results for a three day simulation, where only the key performance indicators of days 2 and 3 have been considered in order to avoid transient responses. Regarding the results, note that the two proposed MPC tuning strategies outperform the equally weighted MPCs in all the indicators. Moreover, the MPC with adaptive weighting combinations has shown the lowest KPIs. Note that the main improvements have been reflected in the KPIs related to dynamical issues of the system rather than in the economic aspects. This fact is due to the absence of management criteria enough to reflect real-system conditions.

\section{CONCLUSIONS}

Reviewing the results, the following observations can be highlighted: the study of the methods based on the normalised normal constraints idea has been useful to find an effective and robust normalisation scheme for the objective functions. The main advantages of the NWS are that the MOO problem remains convex and, hence, the solver time is smaller. In the first DM simulation, it can be seen that the best result is the one obtained with the best trade-off between the objectives (used as baseline for the performance metrics). A better economic result can be obtained but the smoothness is sacrificed. This is a typical behaviour of multi-objective optimisation problems. The histogram-based MPC tuning strategy has proved to work, and it can be used without regrets as a starting point for empirical tuning. The model-based MPC tuning strategy shows the best results. The two presented MPC tuning strategies can be classified as explicit, because they give the weighting factors in an explicit way. It is important to highlight that a compatible online Pareto front calculation, like ENNCP or NWS, together with the DM algorithm constitutes an implicit MPC tuning strategy, because an MPC solution that goes in line with the management criteria is selected at each sampling time, without an explicit calculation of the MPC weighting factors.

\section{REFERENCES}

Adrian, J. (2010). Enhancement of management criteria in the MPC design for Predictive Controllers of drinking water networks. Master's thesis, Universitat Politècnica de Catalunya.

Brdys, M. and Ulanicki, B. (1994). Operational control of water systems: structures, algorithms and applications. Prentice Hall.

Ding, Y., Gregov, S., Grodzevich, O., Halevy, I., Kavazovic, Z., Romanko, O., Seeman, T., Shioda, R., and Youbissi, F. (eds.) (2006). Discussions on normalization and other topics in multiobjective optimization. Fields-MITACS, Fields Industrial Problem Solving Workshop.

Garriga, J.L. and Soroush, M. (2010). Model predictive control tuning methods: a review. Industrial \& Engineering Chemistry Research, 49(8), 3505-3515.
Gatzke, E.P. and Doyle III, F.J. (2001). Model predictive control of a granulation system using soft output constraints and prioritized control objectives. Powder Technology, 121(2-3), 149-158.

Holmström, K., Göran, A.O., and Edvall, M.M. (2009). User's guide for TOMLAB \%. TOMLAB Optimization.

Kerrigan, E. and Maciejowski, J. (2002). Designing model predictive controllers with prioritised constraints and objectives. In Proceedings of IEEE International Symposium on Computer-Aided Control System Design, volume 1, 33-38. Anchorage, AK (USA).

Logist, F., Houska, B., Diehl, M., and Van Impe, J. (2010). Fast pareto set generation for nonlinear optimal control problems with multiple objectives. Structural $\&$ Multidisciplinary Optimization, $42,591-603$.

Logist, F. and Van Impe, J. (2010). Novel insights for multi-objective optimisation in engineering using normal boundary intersection and (enhanced) normalised normal constraint (submitted).

Logist, F., Vallerio, M., and Impe, J.V. (2011). Explicit weight selection procedure for optimal control problems with weighted objectives. In Proceedings of IFAC World Congress, 6p. (accepted).

Logist, F., Van Erdeghem, P., and Van Impe, J. (2009). Efficient deterministic multiple objective optimal control of (bio)chemical processes. Chemical Engineering Science, 64, 2527-2538.

Long, C.E. and Gatzke, E.P. (2005). Model predictive control algorithm for prioritized objective inferential control of unmeasured states using propositional logic. Industrial \& Engineering Chemistry Research, 44(10), 3575-3584.

Maciejowski, J.M. (2001). Predictive control with constraints. Prentice Hall.

Marler, R.T. and Arora, J.S. (2010). The weighted sum method for multi-objective optimization: new insights. Structural \& Multidisciplinary Optimization, 41, 853-862.

Messac, A., Ismail-Yahaya, A., and Mattson, C. (2003). The normalized normal constraint method for generating the pareto frontier. Structural \& Multidisciplinary Optimization, 25, 86-98.

Ocampo-Martinez, C., Puig, V., Cembrano, G., Creus, R., and Minoves, M. (2009). Improving water management efficiency by using optimization-based control strategies: the Barcelona case study. Water Science \& Technology: Water supply, 9(5), 565575 .

Sanchis, J., Martínez, M., Blasco, X., and Salcedo, J.V. (2008). A new perspective on multiobjective optimization by enhanced normalized normal constraint method. Structural \& Multidisciplinary Optimization, 36, 537-546.

Toro, R. (2010). Smart tuning of predictive controllers for drinking water networked systems. Master's thesis, Universitat Politècnica de Catalunya.

Tyler, M.L. and Morari, M. (1999). Propositional logic in control and monitoring problems. Automatica, 35(4), 565 - 582.

Wojsznis, W., Gudaz, J., and Mehta, T. (2003). Practical approach to tuning MPC. ISA Transactions, 42(1), 149-162. 